\title{
Design of 32 Bit Data path Unit of ARM7 Microprocessor using Sub threshold Current Mode Logic
}

\author{
K.A. Jyotsna, P. Satish Kumar, B.K. Madhavi, Sana Bano \\ ${ }^{1,4}$ CVR College of Engineering Hyderabad, Telangana, Indi, ACE Engineering College, Hyderabad, Telangana, \\ India ${ }^{2}$ Sreedevi Women's Engineering College, Hyderabad, Telangana, India
}

Received: 10 $^{\text {th }}$ December 2017, Accepted: 17th January 2018, Published: 28th February 2018

\begin{abstract}
In the present days, Very Large Scale Integration (VLSI) is focused upon designing energy efficient electronic devices. These are nothing but the embedded gadgets whose core is a processor. Advanced RISC Machine (ARM) is one of the families of computer processors which have an reduced instruction set architecture. Current Mode Logic (CML) is one of the low power logics which is used to design circuits with an extended battery life. The circuits built using this technique are capable of giving a dependable and sturdy system. In this paper, the proposed ARM7 microprocessor has been designed using the Sub threshold Current Mode Logic (STCML). All the design blocks operate in the sub threshold region at $0.24 \mathrm{~V}$ power supply. The Data path of ARM7 has been implemented using Cadence Virtuoso Tool in 45nm technology. The results of the blocks have been viewed on Spectra Simulator and the power and delay calculations have been computed. The propound microprocessor is capable of finding place in DSP and other Embedded applications.
\end{abstract}

Keywords: ARM7 microprocessor, CMOS, Current Mode Logic, Data path, RISC processor, STCML, VLSI

\section{Introduction}

power Dissipation is a major issue in most of the VLSI circuits whose cause is increased leakage currents and with the increasing number of transistors per unit area, this becomes more prominent. This might lead to large amount of heatdissipation from the device which may cause its failure. When the low power application of devices is kept in view, RTL level optimization is required.[1] This is essential to obtain portable devices and those which have longevity in terms of battery life.

The CMOS technology contributes to large number of leakage currents in the transistor out of which Sub threshold leakage current is predominant. This leakage current contributes to the major part of the power dissipated by the transistors. Current Mode Logic (CML) also known as Sub threshold Source Coupled Logic (STSCL) helps the digital circuits to be operated in sub threshold region with reduced leakage currents and meager power
consumption.[2],[3]

Microprocessors also known as single chip computers are the elementary units of information systems. Their performance has been driven by the transistor speed, energy scaling and also by the micro architecture gains advances. These advanced micro architectures have stationed transistor integration capacity, pipelining etc. to furnish increasing performance.

ARM microprocessors are the most ubiquitously used Instruction Set architectures in terms of quantity produced. ARM architects the insidious intelligence that is transfiguring our daily experience. In the world, more than $95 \%$ wearable's work on ARM delivering advanced signal processing capabilities. With the integration of ARM and low power logic, energy efficient devices which meet innovative market demands can be developed.

This paper is formed as follows Section II presents the literature review on Current Mode Logic, STCML and ARM7 Section III presents the Implementation of Datapath components of ARM7, Section IV furnishes the Simulation Results of all the Data path blocks of ARM7 in Cadence Virtuoso Tool and also the Comparison of Power and Delay with voltage scaling is presented. Finally, Section V draws the Conclusions for the proposed methods.

\section{A. Traditional CML Topology}

The logic working in an CML gate takes place in the current domain and hence the operating speed is innately high. The Fig. 1, shows a logic network where an source coupled differential pair of NMOS switches, directs the tail current $\left(\mathrm{I}_{\mathrm{SS}}\right)$ to one of the output sections hinging on the logic levels of input. The output load resistance $\left(\mathrm{R}_{\mathrm{L}}\right)$ converts this current to voltage in order to impel the following $\mathrm{CML}$ gates. The output voltage swing $\left(\mathrm{V}_{\mathrm{SW}}=\mathrm{R}_{\mathrm{L}} \times \mathrm{I}_{\mathrm{SS}}\right)$ should be excessive enough to transform completely the input differential pair of the subsequent stage. This output voltage swing is directly proportional to the value of $R_{L}$. Hence, only when the tail current $I_{S S}$ and output impedance is higher than the leakage currents at the junction and load resistance respectively, this circuit can perform appositely 
even in assertively scaled deep submicron technologies. [3]

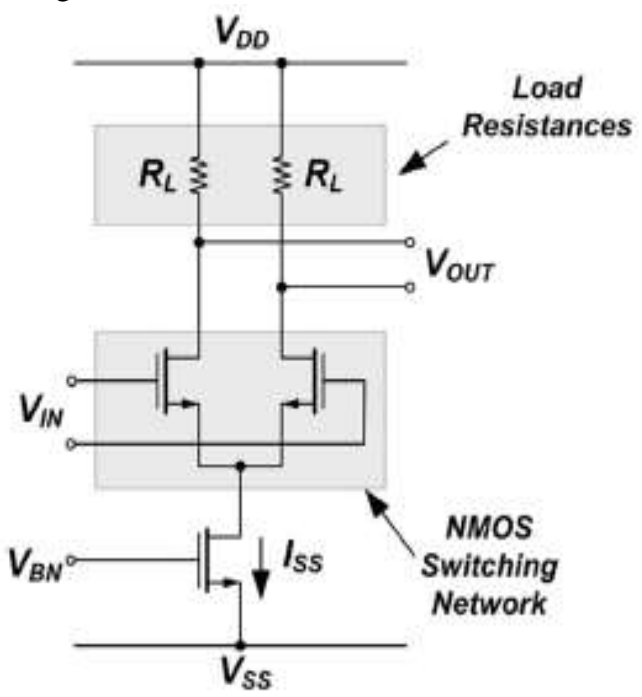

Fig.1 Standard CML Topology based inverter/buffer

The load resistances in the Fig. 1 can be replaced by PMOS transistors operating in triode region. The operating speed in an SCL gate is given by,

$$
\mathrm{T}_{\mathrm{SCL}}=\mathrm{R}_{\mathrm{L}} \times \mathrm{C}_{\mathrm{L}}=\mathrm{V}_{\mathrm{SW}} \cdot \mathrm{C}_{\mathrm{L}} / \mathrm{I}_{\mathrm{SS}}
$$

\section{B. STCML Gates}

In the Sub threshold operation, the tail bias current would be in the range of few nA or less. So, to obtain a reasonable voltage swing at the output, $R_{L}$ should be in the range of $M \Omega$. This resistance can

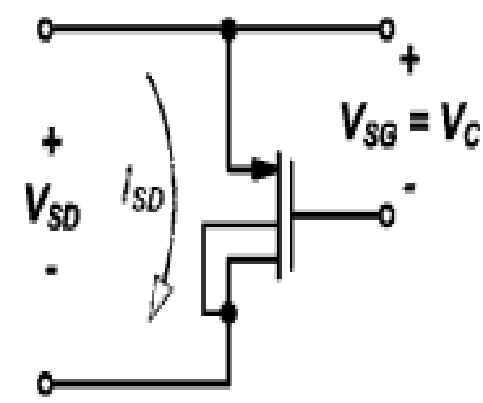

controlled by Iss. Hence, a well-regulated high resistivity device having very small area is required.

Fig.2 PMOS as load device.

Hence, a very high resistivity load device can be implemented using a single PMOS transistor. Since, the devices are of minimum size, the parasitic elements associated. Fig. 2 shows limited and governable differential resistance, which can provide a limited amplitude and gain.[3],[4]. Since, the devices area of minimum size, the parasitic elements associated with the circuit can be ignored. The proposed STCML gate can be used at many operating conditions without having a need for altering the size of transistors. Also, the frequency of operation is directly proportional to the bias current. Fig. 3 shows the circuit of STCML inverter with two gate-drain connected

Fig.3 Circuit of STCML inverter

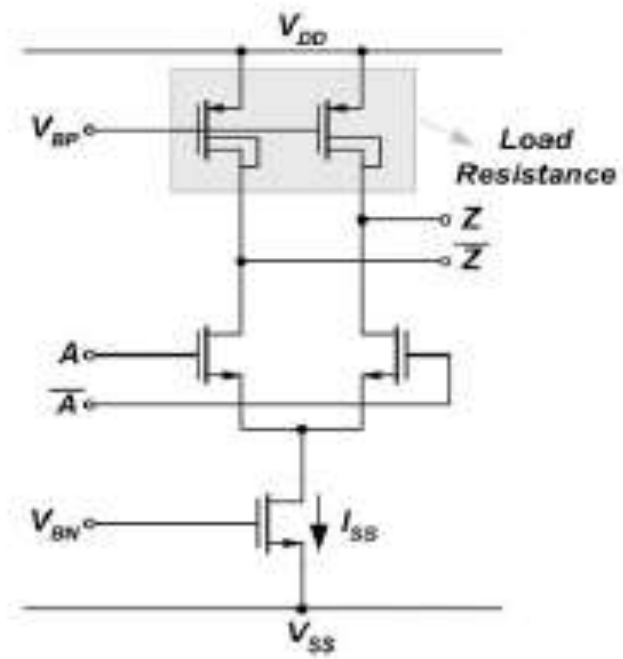

PMOS transistors in the pull-up and three NMOS transistors with one NMOS acting as current source in the pull-down. [4]

Total Delay is given as

$\mathrm{td}=\ln (2) \times \mathrm{C}_{\mathrm{L}} \cdot \mathrm{V}_{\mathrm{sw}} / \mathrm{I}_{\mathrm{SS}}$

The Power-Delay product of the STSCL inverter is given as

$$
\mathrm{PDP}_{\mathrm{STSCL}}=\ln (2) \times \mathrm{C}_{\mathrm{L}} \cdot \mathrm{V}_{\mathrm{SW}} \cdot \mathrm{V}_{\mathrm{DD}}
$$

\section{ARM7 Organization}

The Data path of ARM7 contains Address Register, Address Incrementer, Multiplier, Barrel Shifter, ALU, Write Data Register and Read Data Register all of 32-bit size. It also contains 31 ,32-bit registers in its Register Bank.[5],[6] The Block Diagram showing the Data path is in the Fig. 4. The Register bank of ARM7 can be operated in six modes:

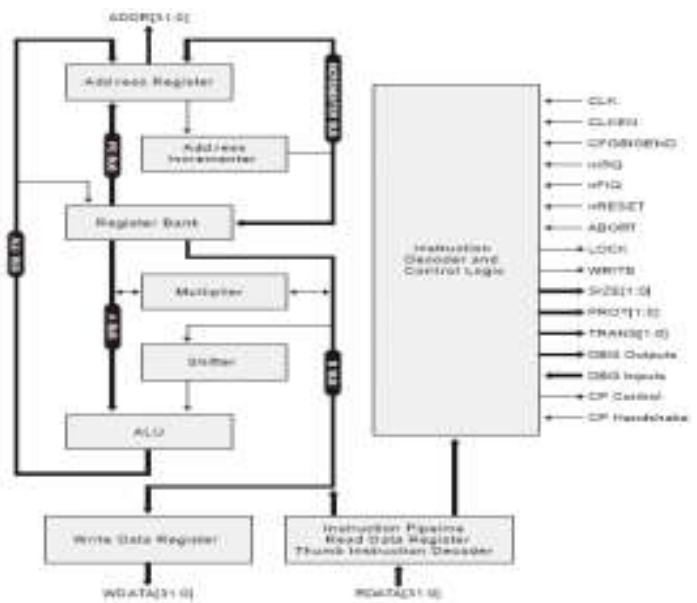

Fig.4 Block Diagram of ARM7 
User mode, Supervisor mode, IRQ mode, FIQ mode, Abort mode and Undefined mode. The multiplier used in this paper for implementation of proposed Data path of ARM7 is $32 \times 32$

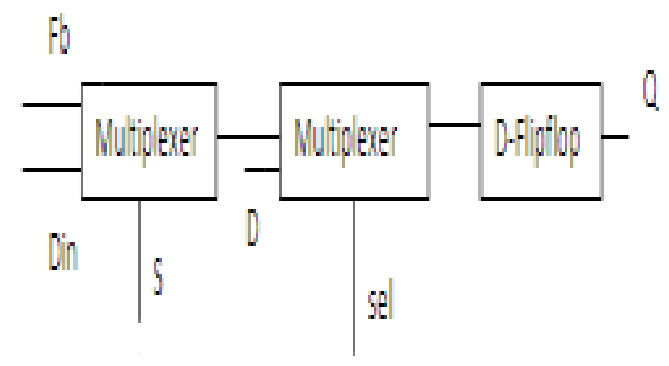

Fig 5 :primary cell of 32 Bit Address Register bit Vedic Multiplier. The ALU is of 32-bit and capable of performing a wide range of Arithmetic and Logic operations[6],[7]

\section{Implementation of Datapath of Arm7 In STCML}

The Datapath 32-bit ARM7 microprocessor has been implemented in Cadence Virtuoso in $45 \mathrm{~nm}$ technology and with the power supply $\left(\mathrm{V}_{\mathrm{DD}}\right)$ of $0.24 \mathrm{~V}$.

\section{A. Address Register}

The Block Diagram of 32-bit Address Register is as shown in Fig.5. The Address Register is used for storing the incremented address of the Program Counter. Its schematic contains Multiplexers and DFlip flops.

\section{B. Arithmetic and Logic Unit}

The Block Diagram of 32-bit Arithmetic and Logic Unit (ALU) is as shown in the Fig.6. The proposed ALU is capable of performing 14 operations on 32bit inputs $a<31: 0>$ and $b<31: 0>$. Table I shows the Arithmetic and Logical operations performed by the proposed ARM7.

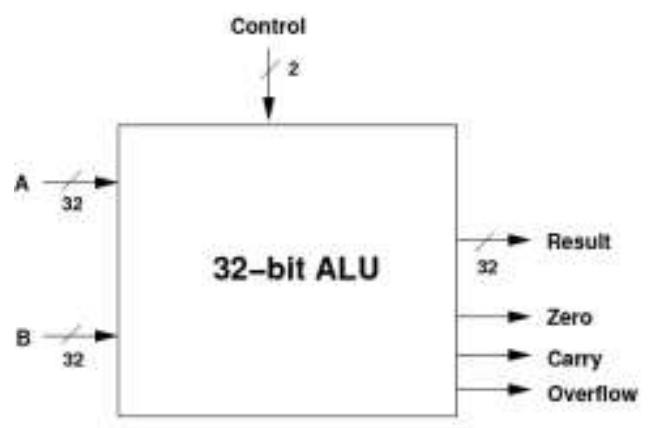

Fig. 6 Block Diagram of 32-bit ALU

The presented 32-bit ALU also sets the Zero, Carry and Overflow flags when the 32-bit result is all 0's, when there is a carry that is propagated or when the overflow condition has occurred. [6]

Table I Operations performed by the proposed 32-bit ALU

\begin{tabular}{|c|c|c|c|}
\hline S1.Xo. & $\begin{array}{c}\text { Select lime } \\
\xi\end{array}$ & Outout & Operation \\
\hline 1 & \multirow{7}{*}{1} & out $1<310\rangle$ & Add Subtract \\
\hline 2 & & out $2\langle 31.0\rangle$ & Logical AND \\
\hline 3 & & out $3\langle 31: 0\rangle$ & Bit Clear \\
\hline 4 & & out $4\langle 31.0\rangle$ & Bit Invert \\
\hline 5 & & out $5<31.0\rangle$ & Increment \\
\hline 6 & & out6 & Shift Left \\
\hline 7 & & out? & Rotate Left \\
\hline 8 & \multirow{7}{*}{0} & out $1<31.0\rangle$ & Logical EXOR \\
\hline 9 & & out $2\langle 31: 0\rangle$ & carry_borrow \\
\hline 10 & & out $3\langle 31.0\rangle$ & Logical OR \\
\hline 11 & & out $+31: 0\rangle$ & Compare if equal \\
\hline 12 & & out $5<31: 0>$ & Pass A imput \\
\hline 13 & & oató & Shift Right \\
\hline 14 & & out 7 & Rotate Right \\
\hline
\end{tabular}

\section{32-bit Barrel Shifter}

The schematic of 4-bit Barrel Shifter and the position of input bits after each shifting is as shown in the Fig.7 and Table II respectively. Barrel Shifter is used for shifting and rotating the input with the

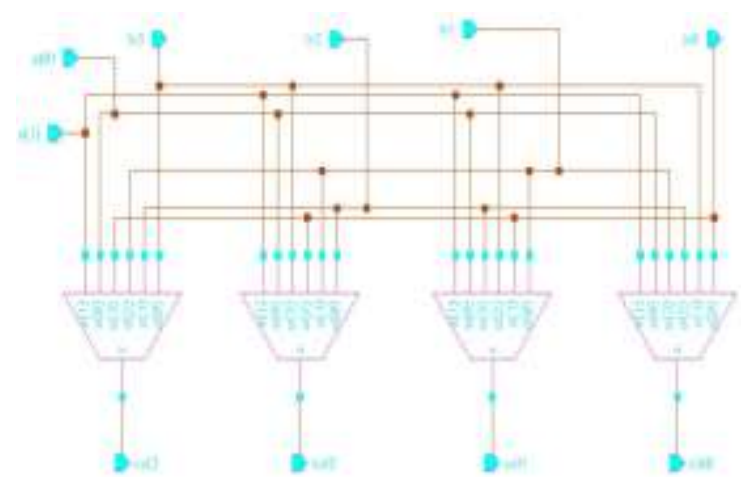

help of Multiplexers. 32-bit Barrel Shifter consists of 32 multiplexers. [5]

Fig.7 Schematic of 4-bit Barrel Shifter

\begin{tabular}{|c|c|c|}
\hline $\begin{array}{l}\text { Select } \\
s_{1} s_{0} \\
\end{array}$ & Operation & $\begin{array}{c}\text { Output } \\
\text { out }_{3} \text { out }_{2} \text { out }_{1} \text { out }_{0} \\
\end{array}$ \\
\hline 00 & No rotation & $i m_{3} i m_{2} i m_{1} i m_{0}$ \\
\hline 01 & Rotate left by 1 bit position & $i m_{2} i m_{1} i m_{0} i m_{3}$ \\
\hline 10 & Rotate left by 2 bit positions & $i m_{1} i m_{0} i m_{3} i m_{2}$ \\
\hline 11 & Rotate left by 3 bit positions & $i n_{0} i i_{3} i i_{2} i n_{1}$ \\
\hline
\end{tabular}

Table II A 4-bit Barrel Shifter Operation The 32-bit Barrel Shifter has been designed using eight 4-bit Barrel Shifters. For rotating 4-bits, four 4x1 Multiplexers have been used. [5] 


\section{User Mode of ARM7}

The User Mode facilitates the user to access all the registers in the Register Bank. It consists of r0-r7 General Purpose Registers and 5 Special Function Registers which are Current Program Status Register (CPSR), Saved Program Status Register (SPSR), Link Register (LR), Program Counter (PC) and Stack Pointer (SP).[6] The schematic of User Mode is shown in the Fig.8.

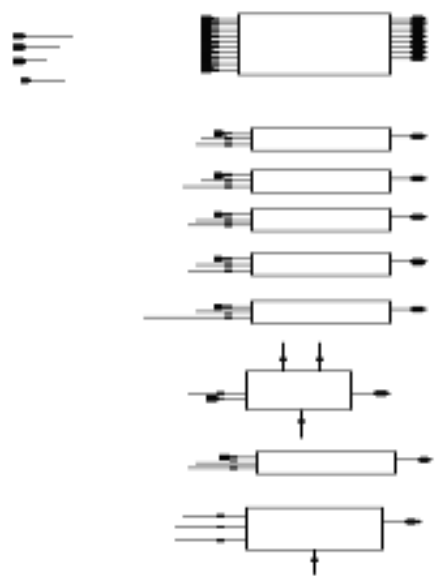

Fig.8 Schematic of User Mode

Also the schematic of 32-bit r0-r7 Registers is shown in the Fig.9. The registers used are 32-bit Parallel-In Parallel-Out Shift Registers (PIPO). All the other modes of ARM7 can be designed similarly by making use of these registers.

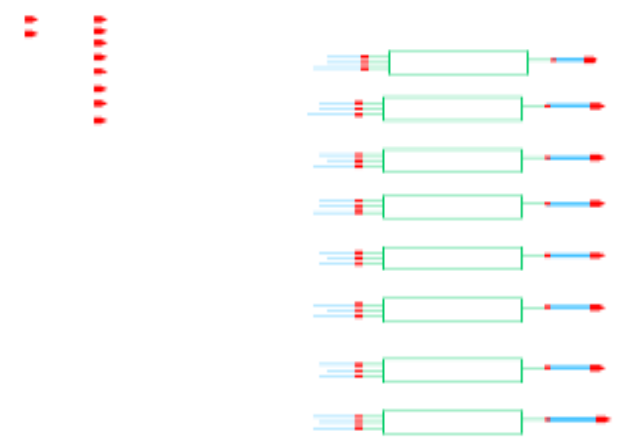

Fig. 9 32-bit r0-r7 general purpose registers

\section{E. 32x32 Vedic Multiplier}

The schematic of $2 \times 2$ Vedic Multiplier is shown in the Fig.10.The Vedic Multiplier is known for its quickness of multiplication and uncomplicated architecture. Not only in the processors, Vedic Multiplier has also been employed for ADSP and DSP applications. The schematic of $32 \times 32$ Vedic Multiplier includes four 16x16 Vedic Multipliers, two 32-bit Ripple Carry Adders and Half Adders.[8]

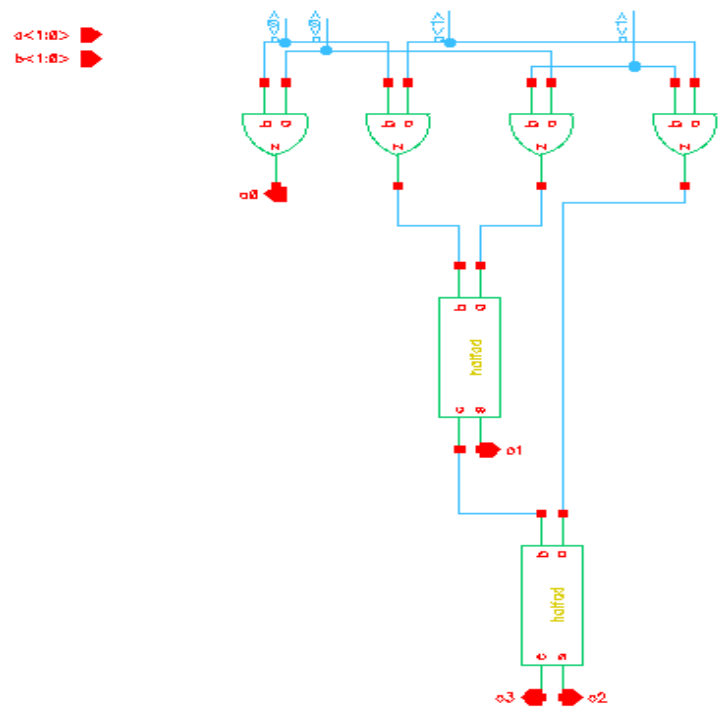

Fig.10 Schematic of 2x2 Vedic Multiplier F. 32-bit Data-In Register

The Block diagram of N-bit Data-In Register is shown in the Fig.11. The 32-bit Data-In Register consists of 32 D-Flipflops connected in Parallel-In Parallel-Out manner.

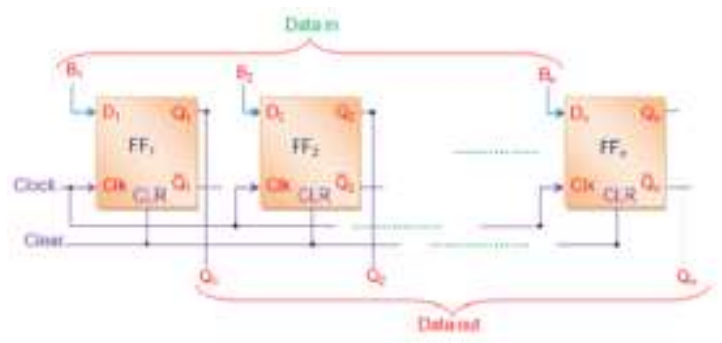

The 32-bit Data-Out register is also designed in the same way like the 32-bit Data-In Register.

G. Data path Integration of ARM7 microprocessor The complete schematic of Data path of ARM7 microprocessor can be viewed in Fig.12.

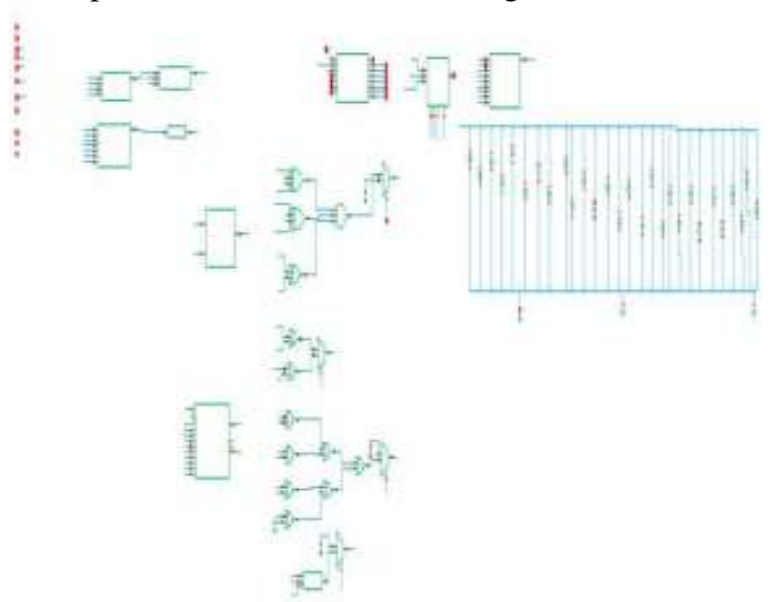

Fig.12: Schematic of Data path of ARM7 microprocessor 


\section{Results}

The simulation results of all the blocks in the Data path of ARM7 have been observed in Spectre simulator. The inputs have a voltage of $0.24 \mathrm{~V}$ as logic high or 1 and $0 \mathrm{~V}$ as logic low or 0 . All the results have been converted from Analog to Digital for ease of examining the output. The results of the Address Register are shown in the Fig.13. The inputs to it are 32-bit address and a feedback. The results of 32-bit ALU are shown in Fig.14 and 15. The outputs can be observed based on the select lines as shown in Table I of this paper. The outputs of the 32-bit Barrel Shifter are shown in Fig. 16. The shifter takes 32-bit input and gives a 32-bit output after shifting and rotate operations are performed.

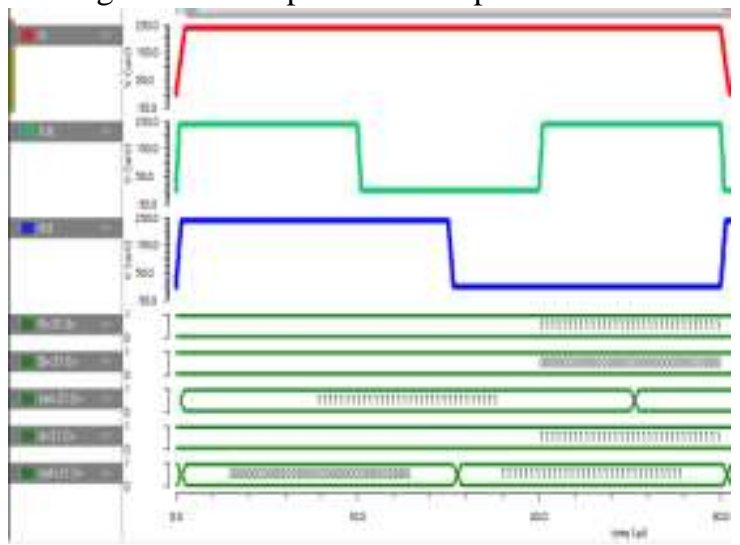

Fig.13: Simulation Results of 32-bit Address Register

When the select line Sel='0', out $1<31: 0>$ gives result of 32-bit addition/ subtraction, out $2<31: 0>$ gives result of Logical AND operation between the inputs $a<31: 0>$ and $b<31: 0>$, out $3<31: 0>$ gives the output of Bit Clear operation, out $4<31: 0>$ gives the result after inverting all the bits of input a $<31: 0>$, out5<31:0> gives the incremented output of input $\mathrm{a}<31: 0>$, out6 and out7 are shift left and rotate left outputs respectively.

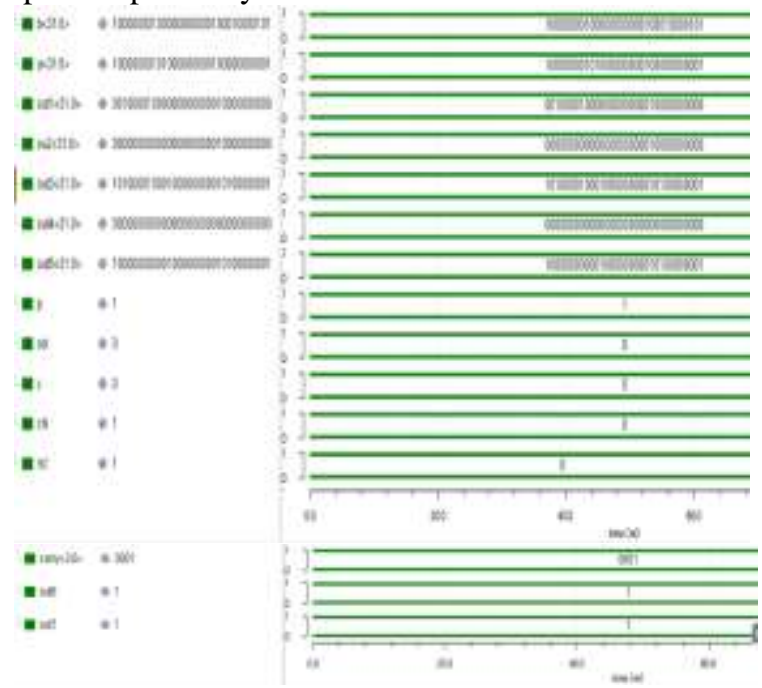

Fig.14: Results of ALU when select line is low
When the select line $\mathrm{Sel}=$ '1', out $<31: 0>$ gives results of Logical EXOR operation performed on inputs $a<31: 0\rangle$ and $b<31: 0\rangle$, out $2<31: 0\rangle$ gives carry/borrow bits of the 32-bit add/subtract operation, out $3\langle 31: 0\rangle$ gives logical OR operation output, out $4\langle 31: 0\rangle$ gives output for equality comparison of $a<31: 0>$ and $b<31: 0>$, for out $5<31: 0>$ passes $a<31: 0>$ as it is, out6 and out7 are the results of shift right and rotate right respectively.

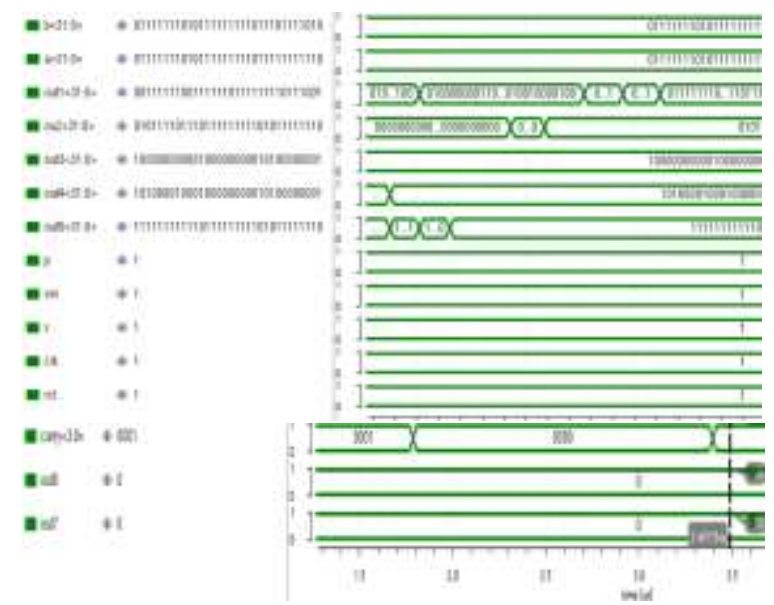

Fig.15 Results of ALU when select line is high.

The outputs of 32-bit Barrel shifter are shown in Fig.16. This shifted output is given to the 32-bit ALU for performing Arithmetic and Logical operations.
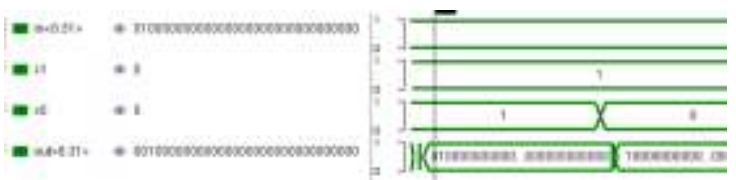

Fig.16 Results of 32-bit Barrel Shifter

The outputs of r0-r7 register bank $\mathrm{z}<0: 255>$ are shown in Fig.17. The common input $\mathrm{D}<0: 255>$ gets shifted into the registers eight registers which are each of 32-bit.

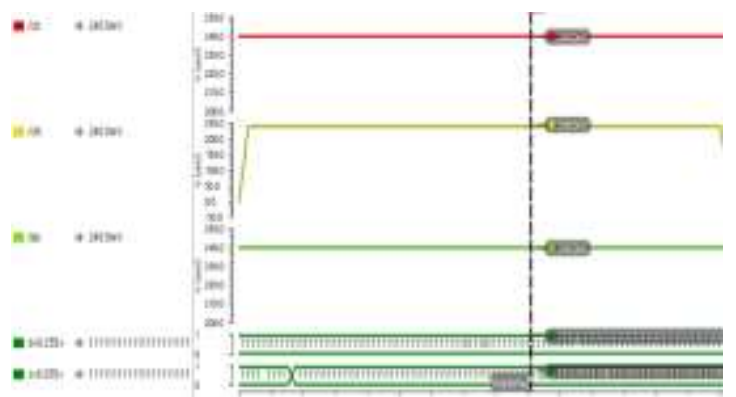

Fig.17 Results of Register Bank 
The output of $32 \times 32$ Vedic Multiplier is as shown in the Fig. 18. The inputs are $a<31: 0>$ and $b<31: 0>$ and the product is $\mathrm{pr}<63: 0>$

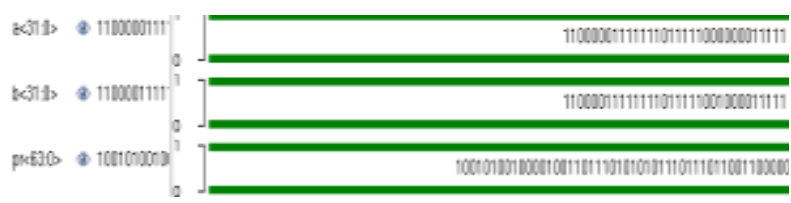

Fig.18 Results of 32x32 Vedic Multiplier

The results of 32-bit Data-In Register are shown in the Fig.19.The input is $\mathrm{D}<31: 0>$ and output is $\mathrm{z}<31: 0>$.

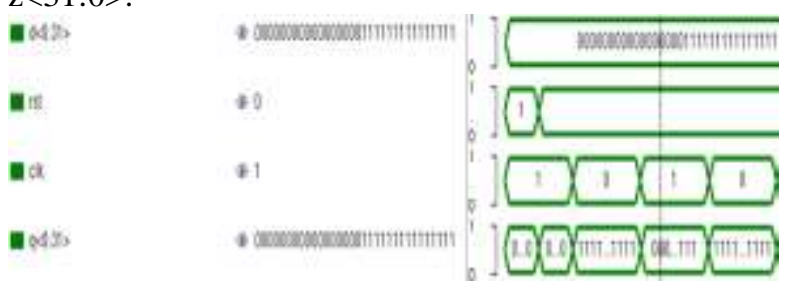

Fig.19: Results of 32-bit Data-In Register

All the Data path components of ARM7 microprocessor have also been simulated at $0.5 \mathrm{~V}$ power supply and the power and delay have been calculated. Table III and Table IV show the comparison of Power and Delay for the Data path components at $0.24 \mathrm{~V}$ and $0.5 \mathrm{~V}$ power supplies respectively.

Table III Power Comparison at Different Power Supplies

\begin{tabular}{|c|c|c|}
\hline Design Block & $\begin{array}{c}\text { STSCL at } 0.24 \mathrm{~V} \\
\text { Power Supply }\end{array}$ & $\begin{array}{c}\text { STSCL at } 0.5 \mathrm{~V} \\
\text { Power Supply }\end{array}$ \\
\hline $\begin{array}{l}\text { 32-bit Barrel } \\
\text { Shifter }\end{array}$ & $18.46 \times 10^{-9} \mathrm{~W}$ & $1.505 \times 10^{-5} \mathrm{~W}$ \\
\hline 32-bit ALU & $172.5 \times 10^{-9} \mathrm{~W}$ & $14.72 \times 10^{-6} \mathrm{~W}$ \\
\hline Register Bank & $479 \times 10^{-9} \mathrm{~W}$ & $43.51 \times 10^{-6} \mathrm{~W}$ \\
\hline $\begin{array}{c}32 \times 32 \text { Vedic } \\
\text { Multiplier }\end{array}$ & $386.4 \times 10^{-9} \mathrm{~W}$ & $78.02 \times 10^{-6} \mathrm{~W}$ \\
\hline $\begin{array}{c}\text { 32-bit Data-In } \\
\text { Register }\end{array}$ & $10.79 \times 10^{-9} \mathrm{~W}$ & $942.8 \times 10^{-5} \mathrm{~W}$ \\
\hline $\begin{array}{c}\text { 32-bit Data-Out } \\
\text { Register }\end{array}$ & $10.68 \times 10^{-9} \mathrm{~W}$ & $940.2 \times 10^{-9} \mathrm{~W}$ \\
\hline $\begin{array}{c}\text { 32-bit Address } \\
\text { Register }\end{array}$ & $23.56 \times 10^{-9} \mathrm{~W}$ & $680.4 \times 10^{-9} \mathrm{~W}$ \\
\hline $\begin{array}{l}\text { 32-bit Address } \\
\text { Incrementer }\end{array}$ & $5.34 \times 10^{-9} \mathrm{~W}$ & $575.4 \times 10^{-9} \mathrm{~W}$ \\
\hline
\end{tabular}

Table IV Delay Comparison at Different Power Supplies

\begin{tabular}{|c|c|c|}
\hline Design Block & $\begin{array}{c}\text { STSCL at 0.24V } \\
\text { Pover Supply }\end{array}$ & $\begin{array}{c}\text { STSCL at 0.5V } \\
\text { Power Supply }\end{array}$ \\
\hline $\begin{array}{c}\text { 32-bit Barrel } \\
\text { Shifter }\end{array}$ & $20.82 u s$ & $20.17 \mathrm{us}$ \\
\hline $\begin{array}{c}\text { 32-bit ALU } \\
\text { Register Bank }\end{array}$ & $25.68 \mathrm{us}$ & $9.79 \mathrm{us}$ \\
\hline $\begin{array}{c}\text { 8-bit RCA } \\
\text { Multiplier }\end{array}$ & $539.1 \mathrm{~ns}$ & $173.4 \mathrm{~ns}$ \\
\hline $\begin{array}{c}32 \text {-bit Data-In } \\
\text { Register }\end{array}$ & $858.4 \mathrm{us}$ & $19.29 \mathrm{us}$ \\
\hline $\begin{array}{c}\text { 32-bit Data-Out } \\
\text { Register }\end{array}$ & $887.5 \mathrm{~ns}$ & $465.2 \mathrm{~ns}$ \\
\hline $\begin{array}{c}32 \text {-bit Address } \\
\text { Register }\end{array}$ & $27.9 u s$ & $359.8 \mathrm{~ns}$ \\
\hline $\begin{array}{c}32 \text {-bit Address } \\
\text { Incrementer }\end{array}$ & $89.2 \mathrm{us}$ & $25.04 \mathrm{us}$ \\
\hline
\end{tabular}

\section{Conclusion}

This paper presents an innovative power efficient methodology for the design of Data path components of ARM7 microprocessor using Cadence Virtuoso in $45 \mathrm{~nm}$ technology with $0.24 \mathrm{~V}$ power supply. It can be inferred from the power calculations that when the voltage scaling is done the power consumption has reduced without causing any effect in the correctness of the waveforms. Also, this method has proved to be efficient for the design of ARM7 and also created room to incorporate the Sub threshold Current Mode Logic with other low power logics in order to reduce the power consumption further if desired.

\section{References}

[1] Sudhakar Alluri, B. Rajendra Naik and N.S.S. Reddy, "Mapping of Five Input Wallace Tree using Cadence Tool for Low Power, Low Area and High Speed," in International Conference on Communication and Signal Processing, April 6-8, 2016.

[2] Armin Tajalli, Elizabeth J. Brauer, Yusuf Leblebici and Eric Vittoz, "Sub threshold Source-Coupled Logic Circuits for UltraLow-Power Applications," in IEEE Journal of Solid-State Circuits, Vol. 43, No.7, July 2008.

[3] M. Horowitz et al., "Low-Power digital design," in Proc. IEEE International Symposium for Low Power Electronics and Design (ISLPED), 1994, pp. 8-11.

[4] Rajiv Gopal and M Murali Krishna, "Subthreshold Design using SCL for Low 
Power Applications," in International Journal of Recent Advances in Engineering \& Technology (IJRAET), ISSN (Online): 2347-2812, Vol.2, Issue 3, 2014.

[5] Enoch O. Hwang, Digital Logic and Microprocessor

Design with VHDL, Brooks/Cole, 2005

[6] Steve Furber, ARM System On Chip Architecture, Addison Wesley, March 2000.

[7] Guen-Young Jeong, Hyun-woo Jo, Byungwoo Yoon and Myung-Jin Lee, "ARM7 Compatible 32-Bit RISC Processor Design and Verification", in $9^{\text {th }}$ Russian-Korean International Symposium (KORUS, 2005), Novosibirsk, Russia, 26 June- 2 July, 2005

[8] Anu Thomas, Ashly Jacob, Serin Shibu and Swathi Sudhakaran, "Comparison of Vedic Multiplier with Conventional Array and Wallace Tree Multiplier", in International Journal of VLSI Design and Communication Systems, ISSN 2322-0929, Vol-4, Issue-4, April 2016, Pages 0244-248 